\title{
Article \\ A Two-Step Handover Strategy for GEO/LEO Heterogeneous Satellite Networks Based on Multi-Attribute Decision Making
}

\author{
Liangyi Zhang $\mathbb{D}^{\mathbb{D}}$, Shaohua $\mathrm{Wu} * \mathbb{C}$, Xiyu Lv $\mathbb{(}$ and Jian Jiao $\mathbb{(}$ \\ Department of Electronic Engineering, Harbin Institute of Technology (Shenzhen), Shenzhen 518055, China; \\ 20s152077@stu.hit.edu.cn (L.Z.); 20S152115@stu.hit.edu.cn (X.L.); jiaojian@hit.edu.cn (J.J.) \\ * Correspondence: hitwush@hit.edu.cn
}

Citation: Zhang, L.; Wu, S.; Lv, X.; Jiao, J. A Two-Step Handover Strategy for GEO/LEO Heterogeneous Satellite Networks Based on Multi-Attribute Decision Making. Electronics 2022, 11, 795. https://doi.org/10.3390/electronics 11050795

Academic Editor: Mauro Tropea

Received: 30 January 2022

Accepted: 1 March 2022

Published: 3 March 2022

Publisher's Note: MDPI stays neutral with regard to jurisdictional claims in published maps and institutional affiliations.

Copyright: (C) 2022 by the authors. Licensee MDPI, Basel, Switzerland. This article is an open access article distributed under the terms and conditions of the Creative Commons Attribution (CC BY) license (https:// creativecommons.org/licenses/by/ $4.0 /)$.

\begin{abstract}
Low Earth Orbit (LEO) satellites can provide high-speed and low-delay services for terrestrial users; however, the rapid movement of LEO satellites and the insufficient size of the LEO constellations incurs the instability of the transmission links. The rapid movement of LEO satellites also leads to frequent handovers. Fortunately, GEO/LEO heterogeneous satellite systems can remedy this shortcoming. As the handover decision strategy which makes the selection among the available satellites will directly impact the performance of GEO/LEO heterogeneous satellite systems, we propose a two-step access and handover decision strategy for heterogeneous satellite networks in this paper. Firstly, a GEO/LEO network selection is carried out based on utility functions that reflect user's QoS requirements. Then, the multi-attribute decision making (MADM) method is used to select the specific LEO satellite if users select LEO satellite network. We also propose an Importance-TOPSIS scheme to improve the weight setting for handover attributes. Simulation results show that the proposed method can reduce the number of handovers and the forced termination probability of the system, and the overall throughput of the system is also improved.
\end{abstract}

Keywords: heterogeneous satellite networks; handover; network selection; multi-attribute decision making; utility function

\section{Introduction}

Due to the limitation of infrastructure construction, the traditional terrestrial mobile network cannot cover some areas, such as remote and non-land regions and stricken areas. The satellite communication system has the advantage of not being limited by geographical environment, so it is an effective solution to provide global coverage. The Geostationary Earth Orbit (GEO) satellites are located 35,786 km above the equator line and move synchronously with the Earth [1]. A GEO satellite covers almost one-third of the Earth's surface [2] (excluding polar regions). Low Earth Orbit (LEO) satellites are generally located at an altitude between $300-1500 \mathrm{~km}$ [1]. Due to the altitude, the inevitable propagation delay of GEO satellite cannot meet the requirements of delaysensitive services [3]. The rapid movement of LEO satellites causes frequent handovers. When users are about to leave the coverage of one LEO satellite, they need to switch to another satellite to avoid communication termination [4]. Moreover, a single LEO satellite covers a small area, and some regions cannot be covered by LEO satellites when the size of the LEO constellation is small.

Subject to issues such as funding and technology, not every country and every institution can provide a large enough LEO constellation. Using small-scale LEO constellations combined with GEO satellites is a feasible and more economical solution. The integration of GEO satellites and LEO satellites can also maximize their respective technological advantages to meet the demands of different types of services. Users need to consider the decision making of handover when they use the GEO/LEO heterogeneous network to communicate. The handover decision is the core stage of the handover process, which 
requires determining the satellite next connected. The newly generated services also need to select the access satellite at the beginning of communication. In order to guarantee user requirements and network load balancing, a rational and practical access and handover decision strategy needs to be proposed to further improve the performance of GEO/LEO heterogeneous satellite systems.

The key point of access and handover decision under multi-satellite coverage is selecting the access point for new calls and handover calls. The decision methods of handover for heterogeneous networks generally include the decision function approach (including simple decision functions [5] and MADM [6]) and the decisions based on complex mathematical models. In [5], authors use the instantaneous received signal strength (RSS) and the average RSS of user equipment to determine the necessity of handover. The simple decision function strategy is easy to implement but does not take full advantage of the information available. The MADM method can effectively and flexibly deal with complex problems [7], so it is often used in heterogeneous networks selection. The Technique for Order Preference by Similarity to Ideal Solution (TOPSIS) method is one of the MADM methods with good performance and wide application. Some researchers also use complex mathematical models to design handover decisions, such as strategies based on the Markov decision process [8], the potential game [9], the graph theory [10], etc. Soft sets, rough sets, and fuzzy sets can also be used in decision making [11]; Riaz et al. introduce the novel concept of the linear Diophantine fuzzy set (LDFS) to help the multi-attributes decision making [12]. These model-based methods are highly accurate, but computationally complex, and have high requirements on the computing power or storage capacity of the device. Considering the limitation of equipment capability, we use TOPSIS to design an access and handover strategy, which can better solve the goal of users to make handover decisions independently.

In this paper, we propose a new strategy to solve the access and handover problems of GEO/LEO heterogeneous satellite networks. The strategy we proposed consists of two steps. Users firstly make the network selection between GEO satellite and LEO satellites using utility functions which consider the communication delays and load status of satellites, then choose an LEO satellite using the Importance-TOPSIS if users select to access the LEO satellite network in step one. In addition, each user does not need to rely on other users' information when making decisions. The simulation results demonstrate the performance of the strategy in system throughput, number of handovers and the forced termination probability.

The main contributions of this paper are as follows:

1. A GEO/LEO heterogeneous satellite network is established to ensure that users can access GEO satellite when the size of LEO constellation is too small to cover the Earth seamlessly. Users can access a GEO satellite or an LEO satellite, and make decisions independently.

2. The utility functions are designed to reflect the demands of services with different delay tolerance for each satellite. The communication delays and load status of each satellite are considered to ascertain the urgency and satisfaction of each service accessing each satellite, so that the load balancing can be improved. The utility functions we design can not only better show the user's demands for a certain attribute, but also drive a normalized value.

3. The Importance-TOPSIS scheme is proposed. The importance of each attribute to the target is calculated by using the continuous replacement method proposed in this paper, which is used as the weight of each attribute to improve the TOPSIS scheme.

The rest of this paper is organized as follows: Section 2 describes the related works about heterogeneous satellite network and handover strategies. In Section 3, we present the system model of GEO/LEO heterogeneous satellite networks and the service model. We present the two-step access and handover strategy based on utility function and the Importance-TOPSIS algorithm in Section 4. Simulation results and analysis is given in Section 5. Finally, the conclusions of this paper are drawn in Section 6. 


\section{Literature Review}

In order to design the access and handover decision strategy of heterogeneous satellite networks, we need to pay attention to the heterogeneous satellite network architecture and handover algorithm. The network architectures of GEO/LEO heterogeneous satellite systems have received wide attention from researchers. In [13], the LEO satellites work as the relay satellites to help communicate between GEO satellites and the ground users. In [14], the LEO satellite networks are set as access layers and the GEO satellites constitute the backbone network as the data center for data storage, processing, routing, and network management. In [15], the GEO satellite forwards the control packages sent by the controller to LEO satellites. However, these architectures do not consider that users have to connect GEO satellites to communicate normally when LEO constellations do not have continuous global coverage. GEO satellites can also help balance the load of LEO constellation to reduce the communication termination of delay-sensitive services that must connect to LEO satellites.

MADA strategies, which are often used in handover decision, can be classified into several groups, including the Simple Additive Weighting (SAW), the Analytic Hierarchy Process (AHP), the Grey Rational Analysis (GRA), the TOPSIS, etc. The SAW method is used in [16] to make the handover decision, this method is simple to implement but poor in accuracy [17]. In [18], the AHP method is used to select the network. The AHP method makes decisions on complex problems by dividing complex problems into a simple and easy-to-analyze decision factor hierarchy. However, AHP requires pairwise comparisons between attributes and the scoring of decision makers, so it needs the expertise of human beings [17]. GRA is also used in the network handover, and the networks can be ranked by calculating the grey relational coefficient [19]. The best solution selected by using the TOPSIS method is the one closest to the ideal solution and furthest from the worst solution [7].

The TOPSIS method needs to set the weight of each attribute. The work of [20] uses AHP to define the weights of the criteria, which needs the subjective judgment of the decision maker in the process. In [21], the analytic network process (ANP) is used to set the weight of attributes. The structure of ANP is more diverse than that of AHP, and it can better describe the structure of complex systems, but it also requires pairwise comparisons between attributes and the scoring of decision makers. The entropy weight method can also be utilized in weight setting [22], which uses the information entropy of attributes. The smaller the information entropy of an attribute is, the larger the weight will be. However, the entropy weight method is lacking in reflecting the contribution and importance of a specific attribute to the target.

Therefore, inspired by the above literature, considering the practical limitations, the two-step access and handover strategy is proposed in this paper, which is based on the background of a heterogeneous satellite network of small-scale LEO satellite constellation combined with GEO satellites, a modified TOPSIS method is used to design a handover decision strategy.

\section{System Model}

As shown in Figure 1, several users (satellite mobile terminals, Internet of Things gateways, vehicle-mounted mobile stations, shipborne mobile stations, etc.) communicate through LEO and GEO satellites. Terrestrial users are covered by a GEO satellite and $n$ LEO satellites, and user's speed is negligible compared to the speed of LEO satellite. GEO and LEO satellites use different frequency bands, and all LEO satellites share the same frequency band. Each user can only collect the information of the covered satellites when deciding, and cannot know the status and decision results of other users, which means the users will make decisions independently.

Due to the rapid movement of LEO satellites, the user may have to handover several times during the communication. When it comes to a new call or a handover call, the user 
must decide which satellite to access, which is the purpose of the solution in this paper. The channel model of satellite-ground link and the service model is described in this section.

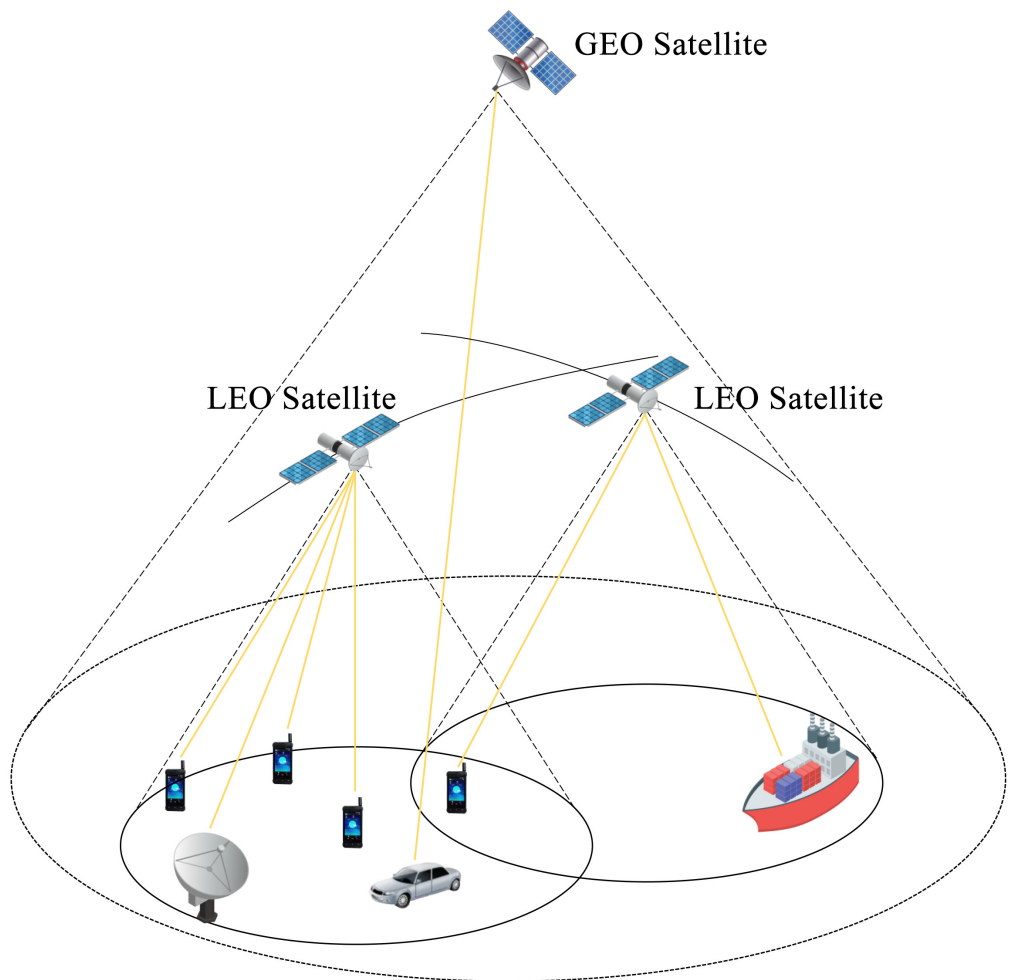

Figure 1. GEO/LEO heterogeneous satellite system.

\subsection{Channel Model}

Radio waves are affected by various factors during propagation, such as free space propagation loss, rain, fog, poor angle of inclination, etc. As satellite-ground links operate at high-frequency bands, atmospheric attenuation will be the major effect for propagation channels. The Shadowed-Rician fading model is widely adopted in the current literature $[23,24]$, and the channel fading coefficient is:

$$
\tilde{h}_{p, q}=A_{1} \exp \left(j \psi_{p, q}\right)+A_{2} \exp \left(j \phi_{p, q}\right)
$$

where $\tilde{h}_{p, q}$ is the fading coefficients of the channel between satellite $q$ and corresponding terminal $p$, including scattered and line-of-vision (LOS) components. $\psi_{p, q} \in[0,2 \pi)$ denotes the stationary random phase, $\phi_{p, q}$ denotes the deterministic phase of LOS component, and $A_{1}$ and $A_{2}$ represent the amplitudes of the scattering component and LOS component, which are independent stationary random processes following Rayleigh and Nakagami-m distributions, respectively.

Let $x_{p, q}$ be the signals transmitted by satellite $q$ and $N$ be the set of satellites. The received signal $y_{p, q}$ at user $p$ can be formulated as

$$
y_{p, q}=\sqrt{P_{p, q} G_{r e}} \tilde{h}_{p, q} x_{p, q}+\sum_{q^{\prime} \in N \backslash q} \sqrt{P_{p, q^{\prime}} G_{r e}} \tilde{h}_{p, q^{\prime}} x_{p, q^{\prime}}+\text { NOISE }_{p},
$$

where $P_{p, q}$ is the transmit power of satellite $q . G_{r e}$ denotes antenna receiving gain. NOISE N $_{p}$ denotes additive Gaussian noise at the receiver $p$, where $\sigma_{p}^{2}$ denotes the corresponding noise power. The first part of $y_{p, q}$ is the useful signal, and the second part of $y_{p, q}$ is the interference from other LEO satellites.

According to (2), the Signal to Interference plus Noise Ratio (SINR) of the link between user $p$ and satellite $q$ can be expressed as $[25,26]$ 


$$
\gamma_{p, q}=\frac{P_{p, q} G_{r e}\left|\tilde{h}_{p, q}\right|^{2}}{\sum_{q^{\prime} \in N \backslash q} P_{p, q^{\prime}} G_{r e}\left|\tilde{h}_{p, q^{\prime}}\right|^{2}+\sigma_{p}^{2}} .
$$

\subsection{Service Model}

The QoS category of services is defined in ITU-T G.1010 [27]. Referring to the description of service delay tolerance in ITU-T, we divide service into four grades, as shown in Table 1. Each grade of services will be processed differently according to the maximum delay tolerance. Services with different delay tolerance have different requirements to access LEO satellites.

Table 1. Service Classification.

\begin{tabular}{cccc}
\hline Grade & $\begin{array}{c}\text { Maximum } \\
\text { Delay Tolerance }\end{array}$ & Example & Satellite to Choose \\
\hline 1 & $\ll 1 \mathrm{~s}$ & Conversational voice, command & LEO Satellite \\
2 & $2 \mathrm{~s}$ & Video messaging, transactions & LEO or GEO Satellite \\
3 & $10 \mathrm{~s}$ & Streaming audio, download image & LEO or GEO Satellite \\
4 & $\gg 10 \mathrm{~s}$ & Fax, background & LEO or GEO Satellite \\
\hline
\end{tabular}

\section{The Proposed Two-Step Access and Handover Strategy}

In the overall strategy, users need to select one satellite to connect from a GEO satellite and several LEO satellites within the coverage. A GEO satellite has higher orbital altitude than LEO satellites, which will lead to a larger propagation delay, so it is not suitable for some services that are more sensitive to delay. Due to the rapid movement of LEO satellites, users may need to switch several times during the communication. Therefore, users need to decide whether to connect to the LEO satellites according to the services delay tolerance and the load status of the LEO constellation. If the user chooses to connect to LEO satellites, the appropriate LEO satellite should be selected according to other factors. So, we propose a two-step access and handover strategy, which consists of the network selection based on utility function and the LEO satellite selection based on the Importance-TOPSIS. Figure 2 shows the process of the two-step access and handover strategy.

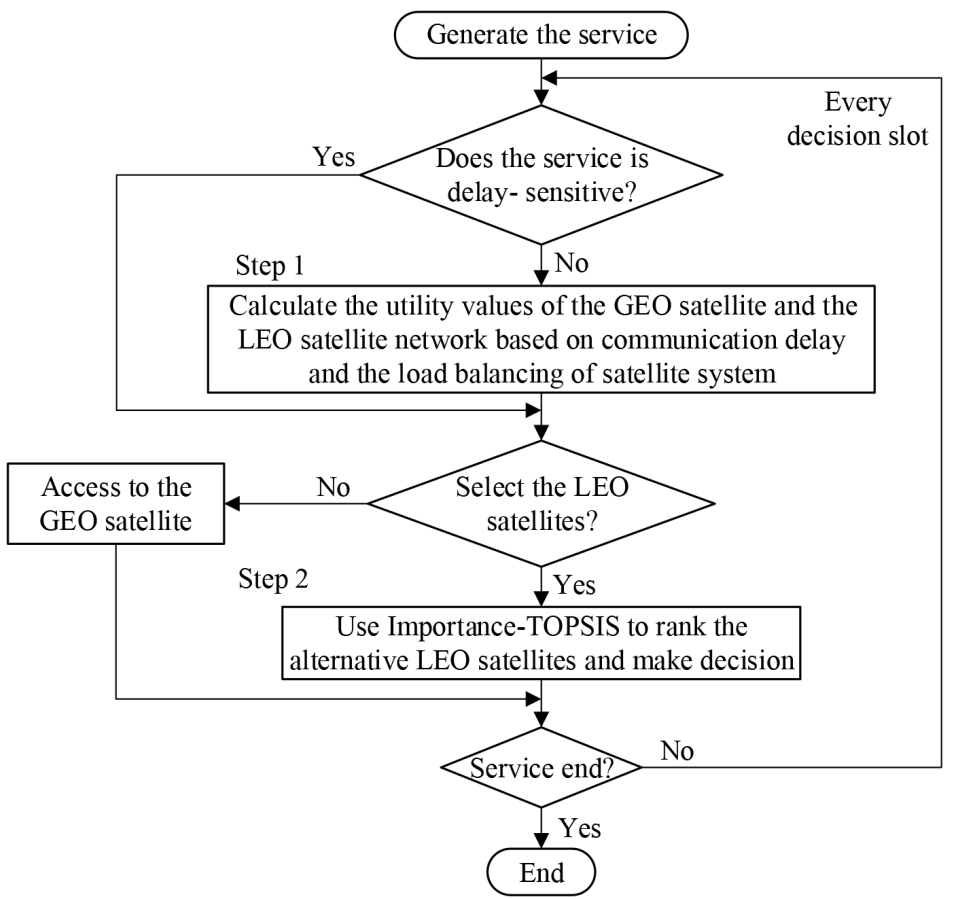

Figure 2. The flowchart of the two-step access and handover strategy. 
As shown in the flowchart (Figure 2), suppose that a mechanical arm working on the sea surface needs to be remotely controlled. The satellites that can be connected to the mechanical arm include one GEO satellite and two LEO satellites. According to Table 1, the control command is in grade 1 service with high delay sensitivity, so the GEO satellite is not considered in the first step. The second step is LEO satellites selection. If the two LEO satellites are not saturated, they would be sorted by using Importance-TOPSIS, and the appropriate LEO satellite is selected for access or handover.

\subsection{Utility Function-Based GEO/LEO Satellite Network Selection}

The first step of the strategy is the selection between the GEO satellite and LEO satellites. The utility function is introduced to process the delay and load status in this step.

In decision making, utility refers to the satisfaction of a commodity or service provided to decision makers [28]. Decision makers with different preferences will have different utility values for the same service. The utility function is a mapping transformation of decision attributes according to attribute utility. In the decision-making design of network selection, the utility function is used to process the decision attributes, which can not only better show the users demands for a certain attribute, but also drive a normalized value [29].

\subsubsection{Utility Design}

Considering the different delay tolerance and different urgency to access the GEO and LEO satellites of services, the design bases of utility function are as follows [29,30]:

- $\quad$ Normalized processing, setting the utility value in the range of $[0,1]$.

- When the communication delay exceeds the service delay tolerance, the utility value of delay is 0 .

- With the increase in delay, the utility value of delay decreases.

- When there is no subchannel available in the satellite, the utility value of availability is 0 ; when all subchannels in the satellite are free, the utility value of availability is 1 .

- As this step is to connect to GEO satellite or LEO satellites, LEO satellites are regarded as a whole.

- Under the same load status, the less the service delay tolerance, the more inclined it is to access the LEO satellite network.

At every time slot $t, d_{i, s}(t)$ is the time delay generated when user $i$ connects satellite $s, r_{i, s}(t)$ is the current available rate of satellite $s$, where $s=1$ is connect to GEO satellite, $s=2$ is connect to LEO satellite network. Letting $d_{t}^{i, j}(t)$ be the transmission delay between user $i$ and satellite $j$ at time $t, d_{p}^{i, j}(t)$ be the propagation delay between user $i$ and satellite $j$, $d_{h o}^{i, j}(t)$ be the handover delay if user $i$ connect to satellite $j$, where $j=1$ is GEO satellite, $U$ is the LEO satellites that can be connected at the current moment.

$d_{i, s}(t)$ is expressed as

$$
d_{i, s}(t)=\left\{\begin{array}{cc}
d_{t}^{i, 1}(t)+d_{p}^{i, 1}(t)+d_{h o}^{i, 1}(t), & , s=1 \\
\frac{1}{|U|} \sum_{j \in U}\left(d_{t}^{i, j}(t)+d_{p}^{i, j}(t)+d_{h o}^{i, j}(t)\right) & , s=2
\end{array}\right.
$$

$r_{i, s}(t)$ is expressed as

$$
r_{i, s}(t)= \begin{cases}\frac{N_{G a}}{N_{G t}} & , s=1 \\ \frac{N_{L a}}{N_{L t}} & , s=2\end{cases}
$$

where $N_{G a}$ is the number of available channels of GEO satellite, $N_{G t}$ is the number of total channels of GEO satellite, $N_{L a}$ is the number of available channels for all LEO satellites that can be connected, and $N_{L t}$ is the number of total channels for all LEO satellites that can be connected. The available channels include free channels and the channel which is already been occupied by user $i$. 
Therefore, at every time slot $t$, the delay utility function $U_{d}^{i, s}(t)$ of user $i$ to satellite $s$ is [29]:

$$
U_{d}^{i, s}(t)=\left\{\begin{array}{cc}
\left.1-e^{\left(k\left(d_{i, s}(t)-d_{i}^{\max }\right)\right.}\right), & 0 \leq d_{i, s}(t) \leq d_{i}^{\max } \\
0 & , d_{i}^{\max }<d_{i, s}(t)
\end{array}\right.
$$

where $d_{i}^{\max }$ is the maximum delay tolerance of the service generated by user $i, k$ is the growth rate, and the higher the value, the steeper the curve. $k$ is determined according to the maximum delay tolerance and the desired delay, namely the Target Point:

$$
k=\frac{-\ln (1-p)}{\left(d_{i}^{\text {max }}-\text { Target Point }\right)}, 0<p<1
$$

where $p$ is inversely proportional to the distance between the Target Point and the tolerance, for most attributes, $0.8<p<0.9$ produces balanced results.

The availability utility function of user $i$ to satellite $s$ at time slot $t$ is [30]

$$
U_{a}^{i, s}(t)=\left\{\begin{array}{cc}
1-\frac{1}{1+e^{15\left(r_{i, s}(t)-\operatorname{Inp}\right)}} & , 0<r_{i, s}(t) \leq 1 \\
0 & , r_{i, s}(t)=0
\end{array}\right.
$$

where the Inp is the inflection point of the curve, which should set according to the maximum delay tolerances so that services with different maximum delay tolerances show different utility values.

Figure $3 a, b$ is the delay utility function and the availability utility function of different grades of services. As the services of grade 1 is time-sensitive and can only connect to LEO satellite, only the services of grade 2,3 , and 4 is calculated.

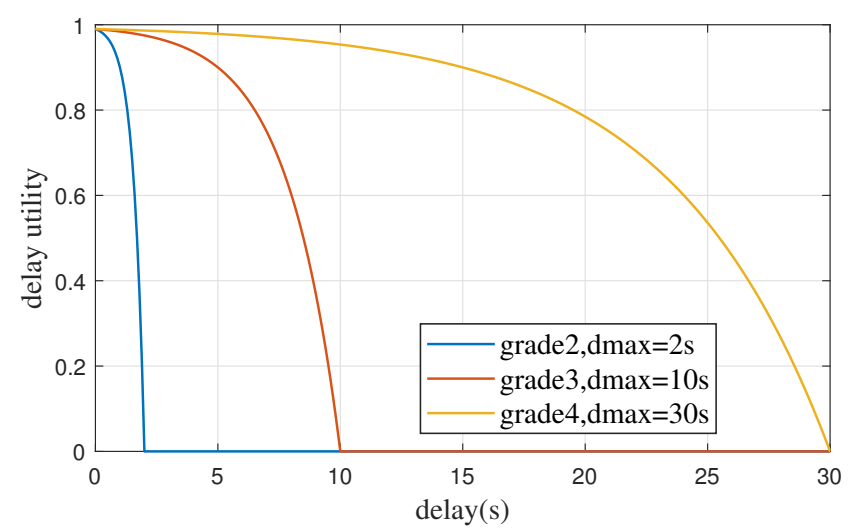

(a)

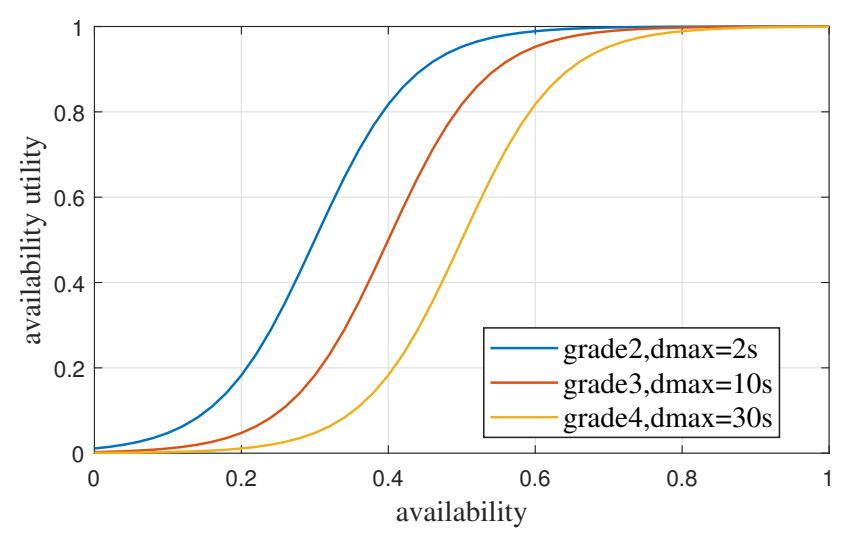

(b)

Figure 3. Utility Function: (a) delay utility function and (b) availability utility function. 


\subsubsection{Problem Formulation}

After obtaining the delay utility function and the availability utility function, the network selection utility function of user $i$ to satellite $s$ is expressed as:

$$
U^{i, s}(t)=U_{d}^{i, s}(t) U_{u}^{i, s}(t)
$$

Letting $x^{i, s}(t)$ be the decision variable, $I$ be the set of users, and function $g(U)$ be set as:

$$
g(U)= \begin{cases}1 & , U>0 \\ 0 & , U=0\end{cases}
$$

the network selection problem is formulated as follows:

$$
\begin{array}{lll}
\max _{x_{i, s}(t)} & \sum_{i=1}^{I} \sum_{s=1}^{2} x_{i, s}(t) U^{i, s}(t) & \\
\text { s.t. } & C 1: x_{i, s}(t) \in\{0,1\} & \forall i \\
& C 2: \sum_{s=1}^{2} x_{i, s}(t) \leq 1 & \forall i \\
& C 3: x_{i, s}(t) \leq g\left(U^{i, s}(t)\right) & \forall i, s
\end{array}
$$

$C 1$ is the constraint of $x^{i, s}(t)$. If $x^{i, s}(t)=1$, user $i$ connect to satellite $s$, while $x^{i, s}(t)=0$ means user $i$ does not connect to satellite s. $C 2$ restricts each user to connect at most one satellite at the same time. The $C 3$ constraints that user $i$ will not connect to satellite $s$ when delay exceeds the tolerance or the satellite $s$ has no available channel.

\subsection{Importance-TOPSIS}

The second step of the decision-making method is when the users choose to connect to LEO satellites, we use a modified TOPSIS scheme to sort the available LEO satellites and select the best satellite to access.

\subsubsection{The Step of Original TOPSIS Scheme}

The steps for TOPSIS are as follows [22,29].

Step 1. Constructing decision matrix. The decision matrix is expressed as

$$
\mathbf{D}=\begin{gathered}
C_{1} \\
\text { Sat }_{1} \\
\text { Sat }_{2} \\
\vdots \\
\text { Sat }_{n}
\end{gathered}\left[\begin{array}{cccc}
d_{11} & d_{12} & \cdots & d_{m} \\
d_{21} & d_{22} & \cdots & d_{2 m} \\
\vdots & \vdots & \vdots & \vdots \\
d_{n 1} & d_{n 2} & \cdots & d_{n m}
\end{array}\right]
$$

where $S a t_{1}, S a t_{2}, \cdots, S a t_{n}$ are the possible alternatives and $C_{1}, C_{2}, \cdots, C_{m}$ are the criteria. Each element $d_{i j}$ of the decision matrix $\mathbf{D}$ is the value of the alternative $S a t_{i}$ with respect to the criterion $C_{j}$.

Step 2. Obtain the normalized decision matrix. The normalized value of $r_{i j}$ is computed as

$$
r_{i j}=d_{i j} /\left(\sum_{i=1}^{n} d_{i j}^{2}\right)^{0.5} ; i=1,2, \cdots, n ; j=1,2, \cdots, m
$$

Step 3. Obtain the weighted normalized decision matrix $v_{i j}$. The weight vector satisfies the following formula:

$$
\omega=\left(\omega_{1}, \omega_{2}, \cdots, \omega_{m}\right), \sum_{j=1}^{m} \omega_{j}=1
$$


After the weights are obtained, each column of the standardized decision matrix is multiplied by its associated weight.

$$
v_{i j}=\omega_{j} r_{i j} ; i=1,2, \cdots, n ; j=1,2, \cdots, m
$$

Step 4. Determine positive and negative ideal solutions.

$$
\begin{aligned}
& Z^{+}=\left(v_{1}^{+}, v_{2}^{+}, \cdots, v_{m}^{+}\right), \text {where } v_{j}^{+}=\left\{\begin{array}{l}
\max \left(v_{i j} \mid i=1,2, \cdots, m\right), j \in I_{b} \\
\min \left(v_{i j} \mid i=1,2, \cdots, m\right), j \in I_{c}
\end{array}\right. \\
& Z^{-}=\left(v_{1}^{-}, v_{2}^{-}, \cdots, v_{m}^{-}\right), \text {where } v_{j}^{-}=\left\{\begin{array}{l}
\min \left(v_{i j} \mid i=1,2, \cdots, m\right), j \in I_{b} \\
\max \left(v_{i j} \mid i=1,2, \cdots, m\right), j \in I_{\mathcal{c}}
\end{array}\right.
\end{aligned}
$$

where $I_{b}$ denotes the set with the benefit criteria, and $I_{c}$ denotes the set with the cost criteria. solution.

Step 5. Calculate the distance of each alternative from the positive (negative) ideal

The distance between the solution and the positive ideal solution is

$$
S_{i}^{+}=\sqrt{\sum_{j=1}^{m}\left(v_{i j}-v_{j}^{+}\right)^{2}} ; i=1,2, \cdots, n
$$

Similarly, the distance between the solution and the negative ideal solution is

$$
S_{i}^{-}=\sqrt{\sum_{j=1}^{m}\left(v_{i j}-v_{j}^{-}\right)^{2}} ; i=1,2, \cdots, n
$$

Step 6. Calculate the relative proximity of each alternative solution to the ideal solution.

$$
T_{i}=\frac{S_{i}^{-}}{S_{i}^{+}+S_{i}^{-}} ; i=1,2, \cdots, n
$$

the optimal satellite is the satellite with the highest $T_{i}$.

\subsubsection{Weight Setting}

As we state in the literature review, in order to overcome the subjectivity and the problem of the existing weight setting methods, this paper intends to propose a new method to define the weight. We use the continuous replacement method to calculate the impact of individual changes of each attribute value on the target value, and give higher weight to attributes that have more impact on the target.

The steps of the continuous replacement method are as follows:

Step 1. Let $(\alpha, \beta) \rightarrow Q,\left(\alpha^{\prime}, \beta^{\prime}\right) \rightarrow Q^{\prime}$, so the difference is $\Delta=Q^{\prime}-Q$. Suppose the order of substitution is to change $\alpha$ first, then change $\beta$.

Step 2. Replace $\alpha$ alone and we find $\left(\alpha^{\prime}, \beta\right) \rightarrow Q_{1}, Q_{1}$ appears due to the change of $\alpha$ on the basis of $Q$. Calculate the influence of $\alpha$ :

$$
Q_{\alpha}=Q_{1}-Q
$$

Step 3. Replace $\beta$ alone and we find $\left(\alpha^{\prime}, \beta^{\prime}\right) \rightarrow Q_{2}, Q_{2}$ appears due to the change of $\beta$ on the basis of $Q_{1}$. Calculate the influence of $\beta$ :

$$
Q_{\beta}=Q_{2}-Q_{1}
$$

We can see that $\Delta=Q^{\prime}-Q=Q_{\alpha}+Q_{\beta}$ 
Step4. Calculate the weight

$$
\omega_{i}=\frac{Q_{i}}{Q^{\prime}-Q^{\prime}} ; i=\alpha, \beta
$$

This method can objectively reflect the importance of each attribute on the target.

\section{Simulation Results and Analysis}

In this section, we evaluate the performance of our proposed access and handover strategy. In the second step, four attributes are used to evaluate the candidate satellites, including SINR, the number of available channels of the satellite, remaining time of satellite coverage, and delay that will exist when connecting the satellite. For comparison, we used the following scheme:

- SINR-max: The decision-making scheme based on SINR. The satellite with the largest SINR will be choosen.

- TOPSIS: TOPSIS scheme without weight setting, which means the weights of all attributes are equal.

- $\quad$ Entropy-TOPSIS: The TOPSIS scheme using the entropy weight method.

- Importance-TOPSIS: The scheme proposed in this paper.

This paper assumes that new calls are generated by independent Poisson arrival distribution, and the duration follows a negative exponential distribution with an average call duration of $600 \mathrm{~s}$. The user makes a decision every $10 \mathrm{~s}$ after the call is generated. The simulation time is $4 \mathrm{~h}$.

\subsection{Simulation Results}

Firstly, we establish the GEO/LEO heterogeneous satellite network constellation model. In order to be more realistic, we use some data from real satellites in the model. The position and coverage of GEO satellites refer to ChinaSat12, and the altitude of LEO satellites is close to that in Iridium satellite system. Parameters of the GEO/LEO heterogeneous network model are shown in Table 2.

Table 2. Satellite System Simulation Parameters.

\begin{tabular}{cc}
\hline Parameters & Value \\
\hline GEO satellite orbital height & $35,786 \mathrm{~km}$ \\
Number of GEO satellites & 1 \\
Bandwidth of GEO satellite & $100 \mathrm{MHz}$ \\
Number of subchannels in GEO satellite & 200 \\
LEO satellite orbital height & $765 \mathrm{~km}$ \\
LEO constellation type & Walker \\
Number of low Earth orbital planes & 6 \\
Number of LEO satellites in orbit & 6 \\
Bandwidth of LEO satellite & $50 \mathrm{MHz}$ \\
Number of subchannels in LEO satellite & 50 \\
Antenna gain & $43.3 \mathrm{dBi}$ \\
G/T parameter & $15 \mathrm{~dB} / \mathrm{K}$ \\
\hline
\end{tabular}

There are various communication scenarios in reality, such as marine environment monitoring, remote control in emergency rescue, Internet services in remote areas, etc. In different communication scenarios, the distribution of various types of services must be different. For example, the proportion of commands in emergency rescue must be larger. To increase the credibility of the results, we generated two distributions of services. The first distribution is that the proportion of delay-sensitive services is small, and the ratio of the quantity of the four services is 1:3:3:3 (grade1:grade2:grade3:grade4). In the second distribution, delay-sensitive services account for a larger proportion, and the ratio of the quantity of the four services is 3:3:2:2 (grade1:grade2:grade3:grade4). Figures 4-6 
respectively show the simulation results of the average throughput, the average number of handovers, and forced termination probability of the system under these two scenarios, (a) the first service distribution of 1:3:3:3 (grade1:grade2:grade3:grade4) and (b) the second service distribution of 3:3:2:2 (grade1:grade2:grade3:grade4).

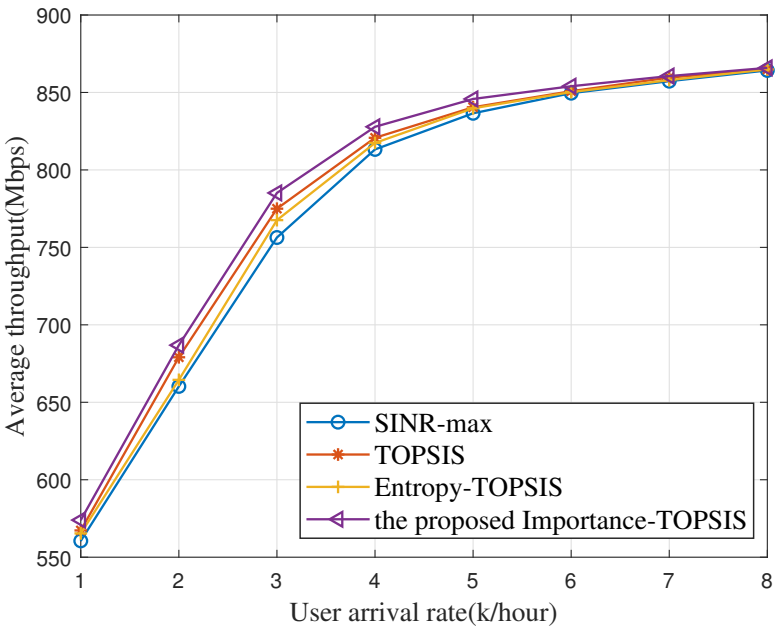

(a)

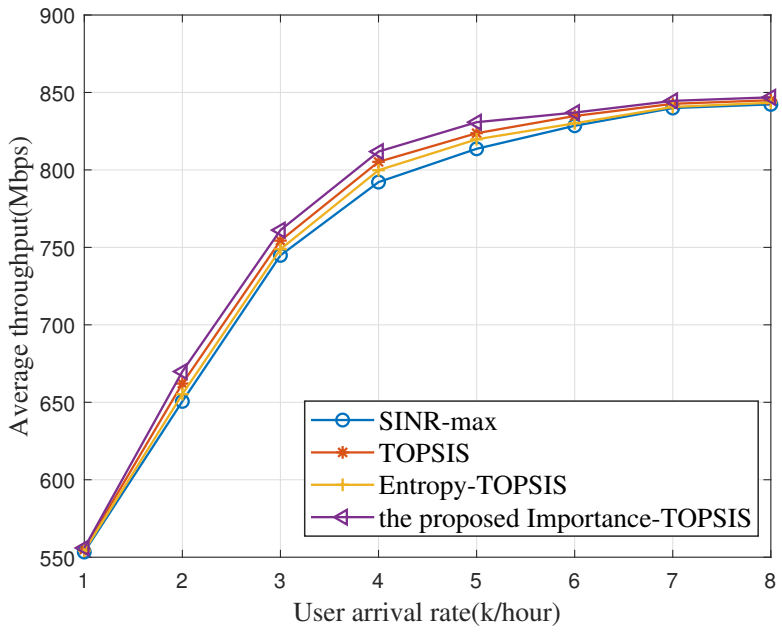

(b)

Figure 4. Average throughput of the satellite system under different schemes. (a) Service distribution 1, (b) Service distribution 2.

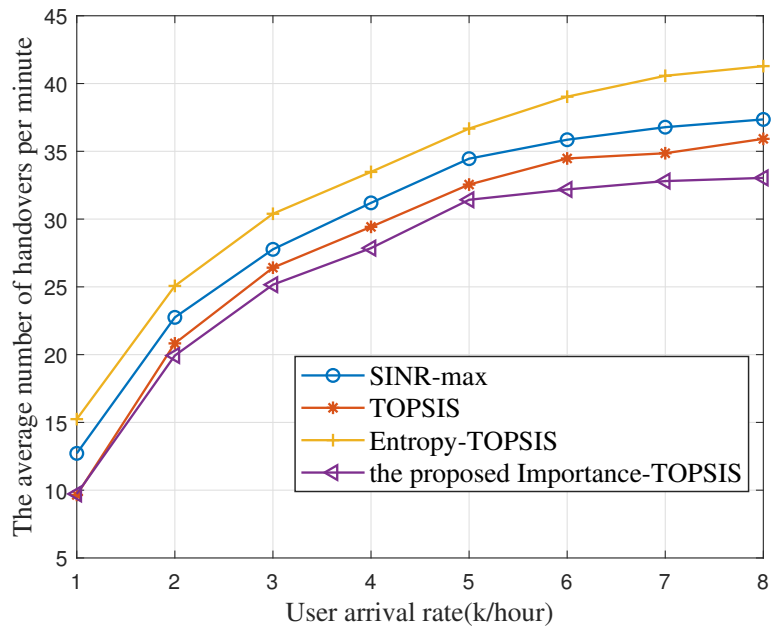

(a)

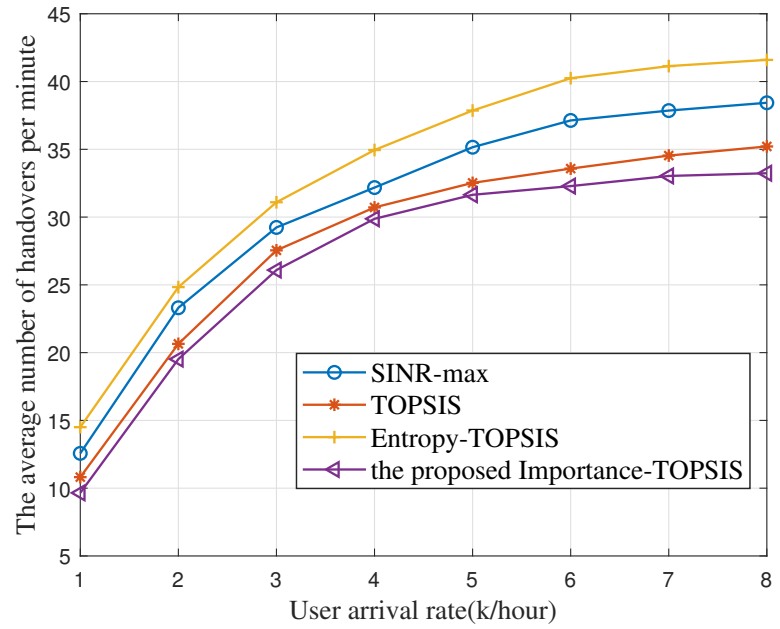

(b)

Figure 5. The number of handovers under different schemes. (a) Service distribution 1, (b) Service distribution 2.

We compare the average throughput of the four schemes under different user arrival rates. As shown in Figure 4, the system tends to be saturated when the user arrival rate reaches 7000 per hour. The Importance-TOPSIS scheme proposed in this paper has the best performance in average throughput, followed by the ordinary TOPSIS scheme with equal weights of all attributes. There is a slight difference between the Entropy-TOPSIS scheme and the SINR-max scheme in throughput, and the entropy weight TOPSIS method is slightly better.

Figure 5 shows the number of handovers per minute at different user arrival rates. It can be seen that the Importance-TOPSIS scheme proposed in this paper has the least average handover times, followed by the ordinary TOPSIS scheme, and the Entropy-TOPSIS has the most average handover times. The SINR-max scheme is more likely to select the 
same satellite in several consecutive selections, and the handover time is less than that of the Entropy-TOPSIS scheme. The Entropy-TOPSIS scheme considers the entropy of each attribute value of candidate satellites when setting the weight. It can be seen that this weight setting method is not helpful in reducing the handover times.

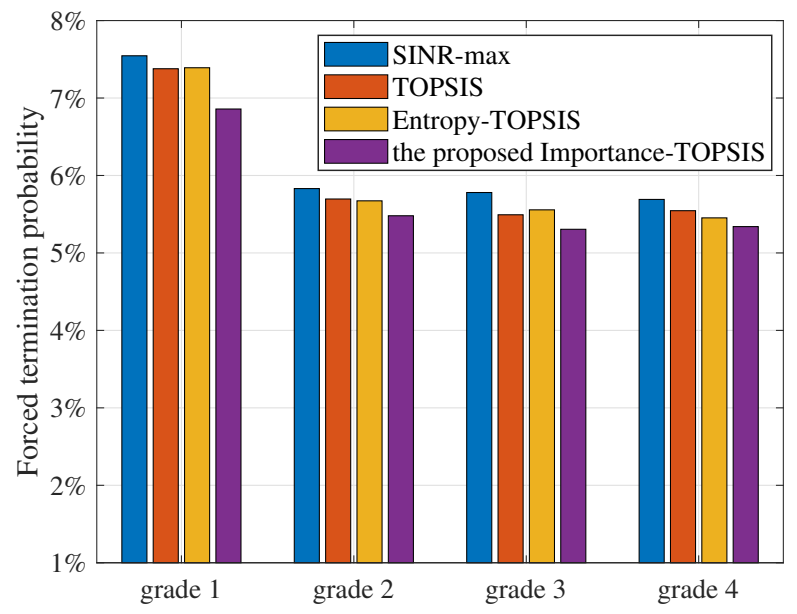

(a)

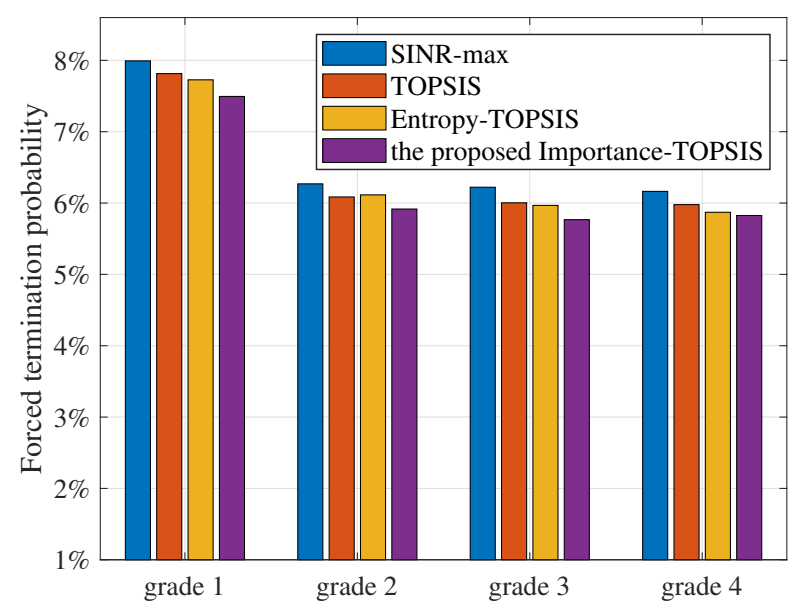

(b)

Figure 6. The number of handovers under different schemes. (a) Service distribution 1, (b) Service distribution 2.

Figure 6 shows the forced termination probability, which is the probability of a call being dropped during the communication of different services grades when the user arrival rate is 5000 per hour. The Importance-TOPSIS scheme has the best performance in the forced termination probability, the other three schemes have similar forced termination probability, and the SINR-max scheme is slightly worse. Combined with Figure 4, part of the reason SINR-max scheme performs worse in system throughput is that the forced termination caused by the load balancing of LEO constellation is not considered. As the services of grade 2, 3, and 4 can connect to the GEO satellite, the probability of forced termination is smaller than that of grade 1.

Comparing the simulation results in different communication scenarios, we find that when the proportion of delay-sensitive services is larger, the system throughput will decrease, and the forced termination probability will increase. This is because delaysensitive services cannot be connected to GEO satellites, a large number of services make the LEO satellite full load, resulting in more access or handover failures, and lower throughput.

\subsection{Sensitivity Analysis}

In Equation (7), there are two controllable parameters, $p$ and Target Point, which are used to change the shape of the delay utility function by adjusting the value of $k$. In order to investigate the sensitivity of these two variables, we simulate the average throughput and the average number of handovers with the changes of $p$ and Target Point.

As shown in Figure 7, the system average throughput and the handover times change with the variation of $p$. In general, the value of $p$ in our method has good results in the range of 0.75-0.85, which is similar to the description in Equation (7). Compared with the results obtained by different strategies in Figures 4 and 5, if $p$ is not in the optimal range, its results may be worse than those of other strategies. 


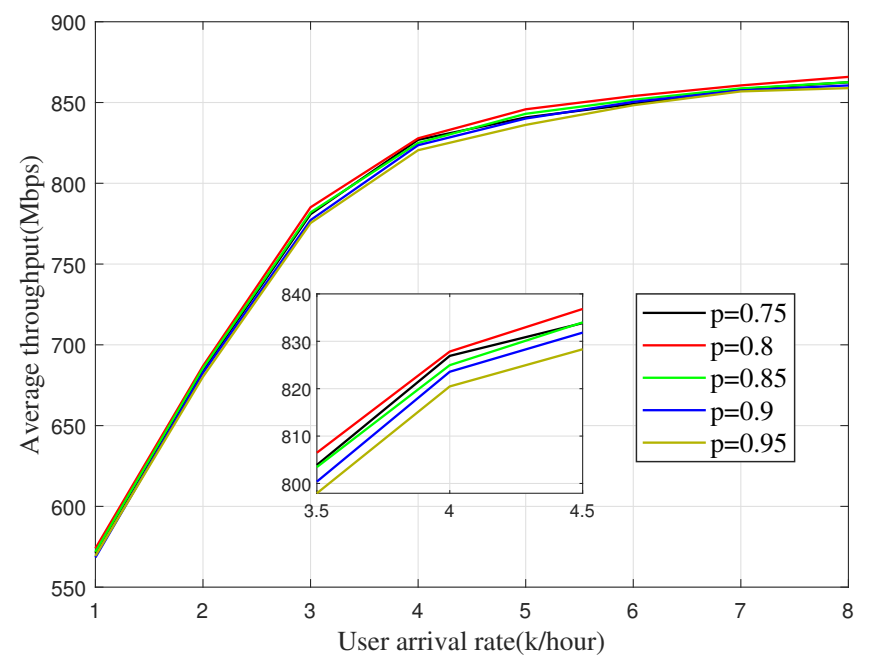

(a)

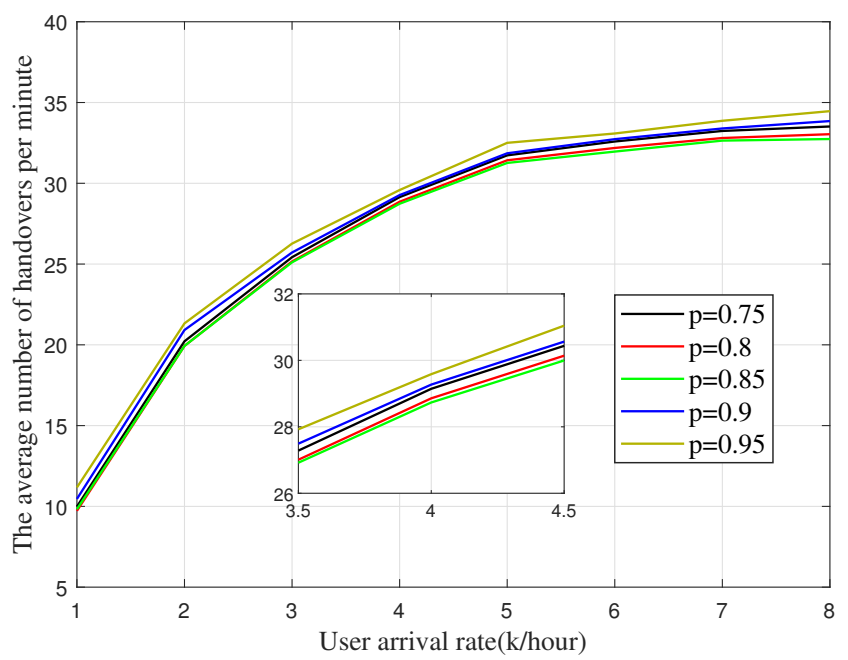

(b)

Figure 7. The sensitivity analysis of $p$. (a) The average throughput, (b) The average number of handovers.

We can know that the value of Target Point cannot exceed maximum delay tolerance from (7). Let the Target Point equals t times maximum delay tolerance, and the simulation results are shown as Figure 8. We can see that the value of Target Point also affects the outcome of the strategy.

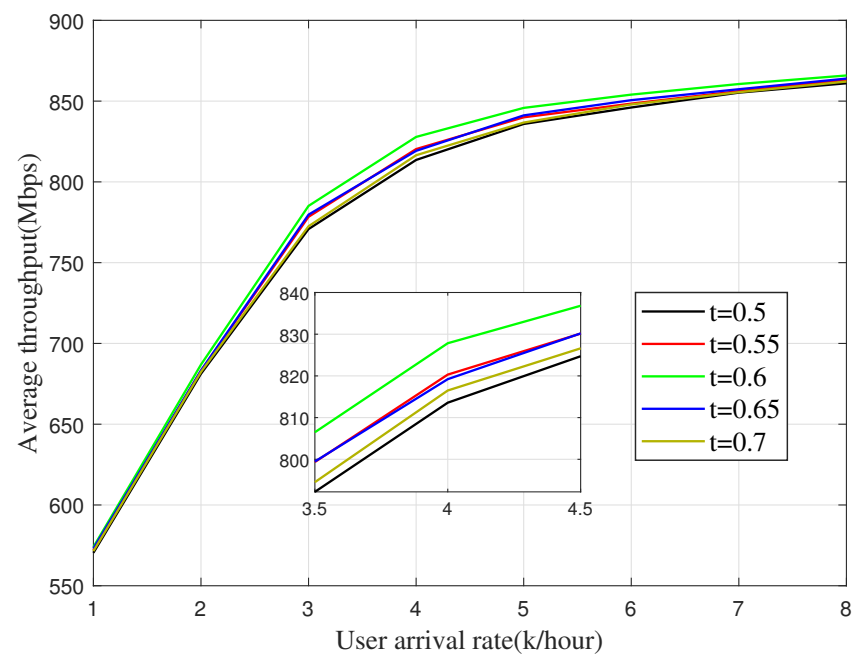

(a)

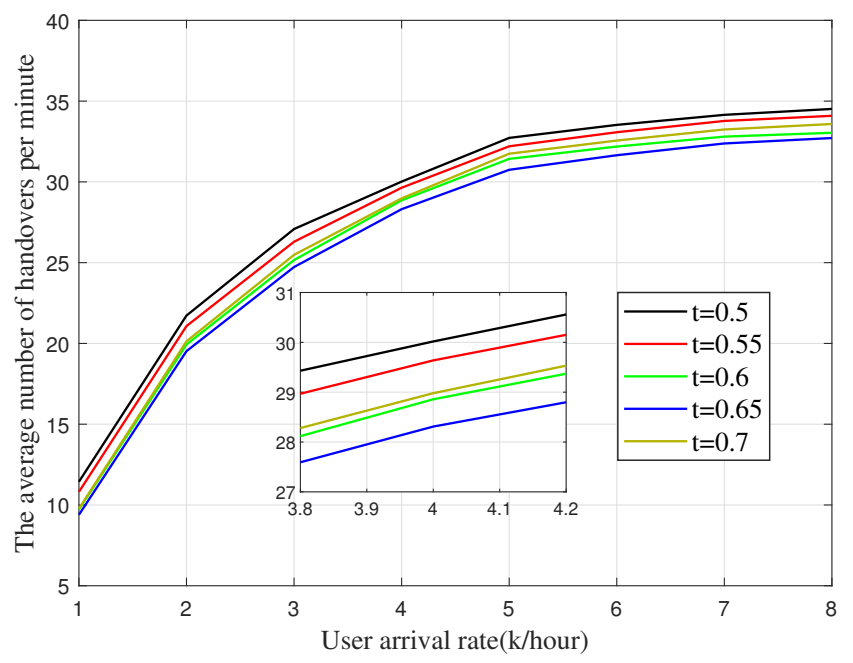

(b)

Figure 8. The sensitivity analysis of Target Point. (a) The average throughput, (b) The average number of handovers.

\section{Conclusions}

The heterogeneous satellite system is the development trend of satellite communication. In order to effectively utilize GEO/LEO heterogeneous satellite network resources, the access and handover decision strategy of the network is particularly important. In this paper, a two-step access and handover strategy for GEO/LEO heterogeneous satellite networks is designed, considering signal quality, handover cost, and load balancing. Firstly, the GEO/LEO satellite network selection function is designed based on the utility function, and then we propose an Importance-TOPSIS scheme to choose an LEO satellite if the users select to access LEO satellites in the first step. Compared with the SINR-max, the original TOPSIS, and the Entropy-TOPSIS handover strategy, the strategy proposed in this paper 
performs better in average throughput, the average number of handovers, and the forced termination probability. The strategy has robustness in a certain degree, and the values of related parameters need to be tested further. We will consider using the fuzzy set and gray method to reduce the robustness of the system for future work.

Author Contributions: Conceptualization, L.Z. and S.W.; methodology, L.Z. and X.L.; software, L.Z. and J.J.; validation, L.Z., S.W. and J.J.; formal analysis, L.Z. and X.L.; investigation, S.W.; resources, L.Z.; data curation, L.Z. and S.W.; writing-original draft preparation, L.Z.; writing-review and editing, L.Z.; visualization, L.Z.; supervision, L.Z.; project administration, L.Z.; funding acquisition, S.W. All authors have read and agreed to the published version of the manuscript.

Funding: This research was funded in part by the National Key Research and Development Program of China under Grant no. 2020YFB1806403, and in part by the National Natural Science Foundation of China under Grant nos. 61871147, 62071141 and in part by the Shenzhen Municipal Science and Technology Plan under Grant no. GXWD20201230155427003-20200730122528002.

Institutional Review Board Statement: Not applicable.

Informed Consent Statement: Not applicable.

Data Availability Statement: Not applicable.

Conflicts of Interest: The authors declare no conflict of interest.

\section{References}

1. Rinaldi, F.; Maattanen, H.L.; Torsner, J.; Pizzi, S.; Andreev, S.; Iera, A.; Koucheryavy, Y.; Araniti, G. Non-Terrestrial Networks in 5G amp; Beyond: A Survey. IEEE Access 2020, 8, 165178-165200. [CrossRef]

2. Chowdhury, P.K.; Atiquzzaman, M.; Ivancic, W. Handover schemes in satellite networks: State-of-the-art and future research directions. IEEE Commun. Surv. Tutor. 2006, 8, 2-14. [CrossRef]

3. Baltaci, A.; Dinc, E.; Ozger, M.; Alabbasi, A.; Cavdar, C.; Schupke, D. A Survey of Wireless Networks for Future Aerial Communications (FACOM). IEEE Commun. Surv. Tutor. 2021, 23, 2833-2884. [CrossRef]

4. Li, J.; Xue, K.; Liu, J.; Zhang, Y. A user-centric handover scheme for ultra-dense LEO satellite networks. IEEE Wirel. Commun. Lett. 2020, 9, 1904-1908. [CrossRef]

5. Yoneya, R.; Mehbodniya, A.; Adachi, F. Two Novel Handover Algorithms with Load Balancing for Heterogeneous Network. In Proceedings of the 2015 IEEE 82nd Vehicular Technology Conference (VTC2015-Fall), Boston, MA, USA, 6-9 September 2015; pp. 1-5. [CrossRef]

6. Lahby, M.; Baghla, S.; Sekkaki, A. Survey and comparison of MADM methods for network selection access in heterogeneous networks. In Proceedings of the 2015 7th International Conference on New Technologies, Mobility and Security (NTMS), Paris, France, 27-29 July 2015; pp. 1-6. [CrossRef]

7. Stamou, A.; Dimitriou, N.; Kontovasilis, K.; Papavassiliou, S. Autonomic Handover Management for Heterogeneous Networks in a Future Internet Context: A Survey. IEEE Commun. Surv. Tutor. 2019, 21, 3274-3297. [CrossRef]

8. Zhou, Z.; Wang, K.; Deng, Z.; Lin, W.; Liu, Y. Switching Algorithm Based On Monte Carlo-Markov Decision Under Space-AirGround Integrated Network. In Proceedings of the 2020 IEEE Wireless Communications and Networking Conference (WCNC), Seoul, Korea, 25-28 May 2020; pp. 1-6. [CrossRef]

9. $\mathrm{Wu}, \mathrm{Y}$.; Hu, G.; Jin, F.; Zu, J. A Satellite Handover Strategy Based on the Potential Game in LEO Satellite Networks. IEEE Access 2019, 7, 133641-133652. [CrossRef]

10. $\mathrm{Hu}, \mathrm{X}$; Song, H.; Liu, S.; Wang, W. Velocity-aware handover prediction in LEO satellite communication networks. Int. J. Satell. Commun. Netw. 2018, 36, 451-459. [CrossRef]

11. Ali, M.I. A note on soft sets, rough soft sets and fuzzy soft sets. Appl. Soft Comput. 2011, 11, 3329-3332.

12. Riaz, M.; Hashmi, M.R. Linear Diophantine fuzzy set and its applications towards multi-attribute decision-making problems. J. Intell. Fuzzy Syst. 2019, 37, 5417-5439. [CrossRef]

13. Bai, L.; Zhu, L.; Zhang, X.; Zhang, W.; Yu, Q. Multi-Satellite Relay Transmission in 5G: Concepts, Techniques, and Challenges. IEEE Netw. 2018, 32, 38-44. [CrossRef]

14. Yao, H.; Wang, L.; Wang, X.; Lu, Z.; Liu, Y. The Space-Terrestrial Integrated Network: An Overview. IEEE Commun. Mag. 2018, 56, 178-185. [CrossRef]

15. Yang, B.; Wu, Y.; Chu, X.; Song, G. Seamless Handover in Software-Defined Satellite Networking. IEEE Commun. Lett. 2016, 20, 1768-1771. [CrossRef]

16. Yu, H.; Ma, Y.; Yu, J. Network Selection Algorithm for Multiservice Multimode Terminals in Heterogeneous Wireless Networks. IEEE Access 2019, 7, 46240-46260. [CrossRef]

17. Li, N.; Zhang, Z.; Liu, A.X.; Yuan, X.; Cheng, Y. Pairwise-Based Multi-Attribute Decision Making Approach for Wireless Network. IEEE/ACM Trans. Netw. 2021, 29, 1687-1702. [CrossRef] 
18. Habbal, A.; Goudar, S.I.; Hassan, S. Context-Aware Radio Access Technology Selection in 5G Ultra Dense Networks. IEEE Access 2017, 5, 6636-6648. [CrossRef]

19. Almutairi, A.F.; Landolsi, M.A.; Al-Hawaj, A.O. Weighting Selection in GRA-based MADM for Vertical Handover in Wireless Networks. In Proceedings of the 2016 UKSim-AMSS 18th International Conference on Computer Modelling and Simulation (UKSim), Cambridge, UK, 6-8 April 2016; pp. 331-336. [CrossRef]

20. Souza, D.D.S.; Vieira, R.F.; Seruffo, M.C.D.R.; Cardoso, D.L. A Novel Heuristic for Handover Priority in Mobile Heterogeneous Networks. IEEE Access 2020, 8, 4043-4050. [CrossRef]

21. Lahby, M.; Cherkaoui, L.; Adib, A. An enhanced-TOPSIS based network selection technique for next generation wireless networks. In Proceedings of the ICT 2013, Casablanca, Morocco, 6-8 May 2013; pp. 1-5.

22. Alhabo, M.; Zhang, L. Multi-Criteria Handover Using Modified Weighted TOPSIS Methods for Heterogeneous Networks. IEEE Access 2018, 6, 40547-40558. [CrossRef]

23. Li, B.; Fei, Z.; Chu, Z.; Zhou, F.; Wong, K.K.; Xiao, P. Robust Chance-Constrained Secure Transmission for Cognitive SatelliteTerrestrial Networks. IEEE Trans. Veh. Technol. 2018, 67, 4208-4219. [CrossRef]

24. Li, B.; Fei, Z.; Xu, X.; Chu, Z. Resource Allocations for Secure Cognitive Satellite-Terrestrial Networks. IEEE Wirel. Commun. Lett. 2018, 7, 78-81. [CrossRef]

25. Cocco, G.; de Cola, T.; Angelone, M.; Katona, Z.; Erl, S. Radio Resource Management Optimization of Flexible Satellite Payloads for DVB-S2 Systems. IEEE Trans. Broadcast. 2018, 64, 266-280. [CrossRef]

26. Cao, Y.; Lien, S.Y.; Liang, Y.C. Deep Reinforcement Learning For Multi-User Access Control in Non-Terrestrial Networks. IEEE Trans. Commun. 2021, 69, 1605-1619. [CrossRef]

27. ITU-T Std. Recommendation G. 1010. End-User Multimedia QoS Categories. 2001. Available online: https://www.itu.int/rec/TREC-G.1010-200111-I/ (accessed on 27 November 2021).

28. Fishburn, P.C. Utility Theory for Decision Making; Technical Report; Research Analysis Corp: McLean, VA, USA, 1970.

29. Senouci, M.A.; Hoceini, S.; Mellouk, A. Utility function-based TOPSIS for network interface selection in Heterogeneous Wireless Networks. In Proceedings of the 2016 IEEE International Conference on Communications (ICC), Kuala Lumpur, Malaysia, 22-27 May 2016; pp. 1-6. [CrossRef]

30. Li, Y.; Zhou, W.; Zhou, S. Forecast Based Handover in an Extensible Multi-Layer LEO Mobile Satellite System. IEEE Access 2020, 8, 42768-42783. [CrossRef] 